\title{
La población indígena de Baja California, México: características físicas
}

\author{
Rose A. Tyson \\ Museo del Hombre de San Diego, California
}

\section{Resumen}

Este artículo consiste en una descripción de los rasgos físicos de los indígenas, tomando como base los relatos de los primeros exploradores que llegaron a la península y los testimonios obtenidos a través de los restos óseos que aún se conservan. De todos los miles de personas que habitaron la península en los últimos milenios, existen sólo aproximadamente 100 individuos representados a través de restos óseos en todos los museos del mundo. Se analizarán muestras de algunos de estos individuos y con el fin de compararlas, se establecerá su asociación arqueológica y su localización en el tiempo. De esta forma, utilizando tanto los relatos históricos como el análisis métrico actual de los restos óseos, surge una más completa descripción de la población prehistórica de la península, de la cual se obtienen resultados interesantes.

Palabras clave: Indígenas de Baja California, restos óseos, características físicas, exploraciones, análisis métrico.

\begin{abstract}
This article describes the physical features of the Indian people. The sources for this research are the narrations that were written by the first explorers who came to the peninsula, and the proofs obtained through bone remains that are still well conserved. From the thousands of people that inhabited the peninsula during the last millenniums, there are approximately 100 individuals who are represented in these bone remains scattered in all the museums of the world. Samples of these individuals will be analyzed and compared in order to establish its archeological association and its location in certain frame of time. In this way, through the analytical use of historical narration such as the current metric analysis of bone remains, we could get a more complete description of pre-historic people of the peninsula, and interesting information.
\end{abstract}

Keywords: Indians of Baja California, bone remains, physical features, explorations, metric analysis. 


\title{
LA POBLACION INDIGENA DE BAJA CALIFOR- NIA, MEXICO: CARACTERISTICAS FISICAS*
}

\author{
Por \\ Rose A. Tyson**
}

\section{INTRODUCCION}

Existen dos métodos para obtener información acerca de las características físicas de los pobladores de la península de Baja California antes de la llegada de los españoles. El primer método yel más subjetivo, consiste en usar las descripciones efectuadas por los primeros exploradores, misioneros y naturalistas. El segundo método de investigación, es la observación directa y el análisis de esqueletos que aún se conservan. Estos dos métodos pueden complementarse y corroborarse el uno al otro.

\section{PRIMERAS DESCRIPCIONES}

Aunque algunos exploradores arribaron a la península durantelos siglos XVI y XVII, pocos dejaron buenas descripciones de la población. Vizcaíno, quien en 1602 inspeccionó la costa del Pacífico, reportó que algunos de los indígenas de cabo San Lucas en el sur, eran pelirrojos. Arriba, más lejos de la costa, en las Balenas, encontró gente de complexión más clara que aquéllas de más al sur (Torquemada, 1966: 241-249).

En 1644 Alonso González, después de una visita al cabo de la península, reportó que la gente era más fuerte y más robusta que la del territorio central de México; sus cabellos eran largos y ligeramente rubios (Martínez, 1956: 109).

El padre Eusebio Kino, quien formó parte de la expedición de Otondo en 1683, reportó a sus superiores que había visto un "cabecilla", quien era "un sujeto muy alto, gigantesco, de aproximadamente 50 años de edad. . ." (Burrus, 1954: 73).

La estatura alta de los indígenas también fue reportada por el padre Salvatierra en sus cartas de 1697 a 1699 desde la misión de Loreto (Burrus, 1971: 113-169).

El capitán Woods Rogers desembarcó cerca del cabo en 1710, encontró cientos de indios, describiéndolos como altos, robustos, rectos, de piel obscura y cabello negro lacio. También hizo comentarios acerca de la avanzada edad de aquéllos que presentaban arrugas en exceso (Rogers, 1966: 355).

\footnotetext{
- Traducido del inglés por Adriana Wells Ayón y Guadalupe Ortega Villa.

* Conservadora de la sección de antropología física del Museo del Hombre de San Diego, California. F.U.A.
} 
Aproximadamente 10 años después, el capitán George Shelvocke visitó esta misma área, y comentó que los hombres eran altos, rectos, bien desarrollados, con extremidades fuertes y pelo negro corto y áspero. Las mujeres eran mucho más pequeñas de estatura, con pelo largo casi cubriendo sus caras. Relató que las personas de ambos sexos tenían caras hermosas y piel color cobre obscuro. También comentó sobre la edad extrema de muchos de los indígenas de ambos sexos, ya que tenían pelo gris y bastantes arrugas (Shelvocke, 1928: 223-227).

En 1721, cerca de 200 años después de que los primeros europeos visitaran la península, el padre Nápoli escribió sobre los indios cora de la región del cabo.

No he visto indígenas más altos que éstos. Son bien proporcionados, corpulentos, y de una constitución muy rojiza [sic]. Los niños especialmente, parecían ingleses o flamencos por su piel clara y rojiza. Es mi creencia, que algunos de ellos quienes son visiblemente diferentes del resto, son hijos de ingleses, ya que por este cabo han pasado muchas embarcaciones inglesas y se han detenido aquí en espera del galeón de Manila (Moriarty, 1970: 55).

El padre Nápoli manifestó también que muchos hombres y mujeres llegaban a la vejez y que incluso había un individuo de más de 100 años de edad. Otro indígena descrito por él, un hechicero, era alto, obeso y con bigote largo (Moriarty \& Smith, 1970: 50-51).

En 1771, el padre Baegert publicó una excelente descripción de San Luis Gonzaga, territorio de los indígenas guaycuras. El reportó una variedad en colores de piel, desde café castaño obscuro, café profundo y rojo cobrizo, hasta casi el negro. Su peloera lacio y negro, sus caras lampiñas, y de cejas escasas. Sus ojos tenían forma de arcos, y en sus ángulos internos eran más bien redondos que puntiagudos. La estatura también era variada, desde altos a bajos, pero todos con postura recta, incluso los ancianos. Los niños podían caminar antes del año de edad. Enfatizó que ninguno era obeso (Baegert, 1952: 53).

Clavijero y Venegas, destacados historiadores, no visitaron la península, pero recopilaron cartas y diarios de muchos misioneros y exploradores. Clavijero escribió a finales de la década de 1780 , las siguientes descripciones resumidas:

Los californianos se asemejan a la gente de México en sus rasgos faciales, pelo, barba y color. Al igual que ellos tienen el cabello grueso, lacio y negro; barba escasa; brazos, muslos y piernas sin vellos; de frente angosta, de nariz un poco gruesa, de dientes blancos, uniformes y fuertes; y boca, ojos y orejas de tamaño regular, excepto aquéllos que se criaron salvajes y deformaron sus narices y orejas con aretes que se colocaban como adornos. El color de la piel de los que viven en lugares tierra adentro es castaño, pero los que habitan regularmente en las playas son más morenos. Las personas deformes son tan raras entre los californianos como entre los mexicanos (Clavijero, 1971: 90) 
Una excelente descripción fue traducida por Aschmann de un manuscrito jesuita que él atribuye al padre Norberto Ducrue, escrito a mediados de la década de 1750 (Aschmann, 1966: 20):

. . . las tribus son realmente muy diferentes unas de las otras en cuanto a su naturaleza, forma y disposición de sus cuerpos. El pueblo pericú que habitaba el extremo sur de la península era antiguamente numeroso, sin embargo, en la actualidad se reduce a un número pequeño y supera a los otros en su buena apariencia. Ellos son generalmente de estatura alta, de buena apariencia y de color más claro... que los otros dos (Aschmann, 1966: 68).

El jesuita continúa su relato al describir a los guaycuras como de color más obscuro y de estaturà mediana, pero robustos y vigorosos (Aschmann 1966: 69-70). También describe a la gente del área del desierto central: "La. . . tribu cochimí, que es la última y la más extendida de todas las que hasta ahora han sido descubiertas, es en general de estatura mediana e incluso pequeña comparadacon las otras tribus" (Aschmann, 1966: 70).

Los primeros 250 años que fomentaron las descripciones antes mencionadas se llevaron a cabo principalmente en 1a parte sur de la peninsula e involucraron a exploradores y misioneros. En los escritos del padre Luis Sales, misionero dominico, son pocas las referencias que describen la parte norte de la península; sin embargo, a finales de la década de 1700 escribió desde la misión de San Miguel, y comenta acerca del color de la piel de la gente: "Todos son de color café intenso, no obstante, hay ciertos indigenas casi blancos, pero creo que esto algunas veces se debe a la mezcla de razas. . ." (Sales 1956: 30). Hohenthal (1951) cree que la gente descrita por Sales era kiliwa, ya que la descripción cultural es muy parecida a aquélla dada por Meigs en su monografía (Meigs, 1939).

A finales del siglo XVIII una nueva ola de exploradores llegó a la península: eran los científicos enviados para describir las tierras recientemente descubiertas.

El primer grupo en explorar la península fue la Real Expedición Botánica de España, 1791-1792, con José Longinos Martínez como naturalista (Simpson: 1961: 58), quien viajó a lo largo de la península y continuó hasta San Francisco en Alta California. Sus observaciones sobre los indigenas de ambas Californias son interesantes. Longinos comentó acerca de la constante disminución de la población de la partesur de la península, la cual estaba siendodiezmada por las enfermedades europeas:

... en tan poco tiempo que tiene de haber sido conquistada, el número de paganos(indigenas) ha disminuido a tal grado que [de] las numerosas tribus. .. que habitaban el distrito del sur, quedan no más de 30 gentes actualmente en las misiones de ese territorio (Simpson, 1961: 21).

Longinos también informó acerca de los indigenas del área norte, misma que fue llamada La Frontera: 
Ellos son bastante grandes, bien formados, fuertes, no muy feos, sin pelo en sus barbillas o en cualquier otra parte de sus cuerpos, salvo en sus cabezas. Esto no se debe a que ellos nazcan así, sino que cuidadosamente se quitan vello por vello tan pronto como aparece, ayudándose de una pequeña vara. . . Los habitantes de la costa hacen uso de ciertas conchas bivalvas, las de mejor forma y fineza, para usarlas como pequeñas pinzas, y de esta manera no dejan cabello alguno en sus cuerpos (Simpson, 1961: 37-38).

Posteriormente Longinos escribió que los indígenas de la región fronteriza se parecian mucho a aquéllos que él conoció después en la parte sur de la Alta California (Simpson, 1961: 58).

Durante los siguientes 100 años, otras expediciones científicas exploraron la península. William Gabb, del Buró de Etnología Americana, hizo una comparación del vocabulario de los cochimi en San Borja y Santa Gertrudis, y el de los kiliwa cerca de San Quintín. Escribió que los cochimí estaban a punto de extinguirse cuantificándolos por debajo de una docena de personas (Gabb, 1867).

La expedición más significativa para el estudio de la prehistoria fue el trabajode Herman Ten Kate, de la Sociedad de Antropología de París. El fue el primero en reportar la existencia de restos fúnebres de esqueletos dolicocéfalos envueltos y pintados de rojo en la región del cabo(Ten Kate, 1883:321-326 y 1884: 551-569). A principios de la década de 1880 , Kate exploró la región del cabo con el naturalista americano Lyman Belding, a fin de descubrir los restos físicos y culturales de los habitantes aborígenes. Otro objetivo de su viaje fue encontrar sobrevivientes de la población indigena primitiva. En el rancho San Jacinto, región del cabo, encontraron a una mujer de aproximadamente 75 años de edad, quien tenía fama de ser de ascendencia indígena. "Ella difería bastante de los yaquis y de otros indígenas de la parte Este del golfo; era de buena estatura, de forma robusta y piel morena, con un cráneo parecido a aquéllos encontrados en las cuevas" (Belding, 1985: 21).

En Todos Santos, en la parte sur de la península, Ten Kate encontró a un hombre llamado Concha, quien también fue reportado como perteneciente a la población originaria. El rechazó ser medido, pero permitió que su color de piel fuera comparado en un cuadro clasificatorio de colores, y aceptó que se le tomara una muestra de su pelo ondulado. Era de estatura mediana, de brazos delgados pero fuertes y de piernas encorvadas (Ten Kate, 1884: 566).

León Diguet efectuó varios viajes a la peninsula durante los años de 1893 a 1905 con el propósito de recolectar restos óseos. También fotografió y midió varios restos de los indígenas cochimí que vivían en el área de Santa Gertrudis (Diguet, 1898).

En 1908, Arthur North viajó hacia el norte de Baja California, donde todavía vivían miembros de las tribus kiliwa, pai pai, kumiai y cucapá. Comentó sobre los rasgos físicos lo siguiente: 
En lo físico, los miembros de todas estas tribus, exceptuando a los diegueños (kumiai), sobrepasaron a aquéllos de la parte sur de Baja California. Yo he visto kiliwas y pai pai de más de seis pies de altura y de proporciones magníficas (North, 1908: 240).

North diferenció dos tipos entre los cucapá: unos de estatura mediana, culor obscuro y constitución fornida; los otros, de tipo menos común, eran más altos y más delgados, de facciones mejor parecidas y un color de piel rojo vivo (North, 1908: 240).

Chittenden también recorrió el norte de Baja California durante el transcurso del siglo XX, y describió a dos cazadores cucapá de quienes comentó que eran más altos que el promedio del nativo americano, fuertes y bien formados, de rasgos regulares, ojos largos y bien abiertos y frentes prominentes (Chittenden, 1901: 201).

\section{DISCUSION DEL MATERIAL DESCRIPTIVO}

La descripción visual que nos llega de los escritos históricos tiene un tema en común: gente alta, de buena constitución, con colores de piel que varían desde el cobre hasta el café obscuro; rectos y de cabello negro lacio. Otras descripciones incluyen lo siguiente: mujeres mucho más pequeñas que los hombres, frentes estrechas, nariz ancha, ojos muy abiertos y además presentaban arrugas. Un comentario en el que todos coinciden se refiere a la edad avanzada de muchos de estos indigenas, misma que se evidenciaba a través del pelo gris y las arrugas en la piel. Algunos de los informes mencionan piel y cabellos claros. Vizcaíno en 1602 vio indígenas pelirrojos en el lejano sur de la península. Debido a que los exploradores tuvieron contacto desde 1534 con los aborígenes, probablemente éste sea el resultado de la mezcla que pudo ser vista en 1602 .

En la época en que estas últimas narraciones fueron hechas, los indígenas de la península se encontraban bajo una tensión extrema, tanto física como cultural. Las enfermedades europeas a las cuales ellos no eran inmunes diezmaron grupos enteros. La vida de las misiones desorganizó las redes sociales y familiares hasta el punto de la descomposición cultural indígena (Aschmann, 1966). Una dieta europea y además una mala alimentación, contribuyeron al debilitamiento de los grupos hasta que las áreas sur y central fueron completamente despobladas. Sólo en el norte, donde las misiones tenían menos control, la población fue capaz de sobrevivir a la llegada de los europeos.

\section{LOS RECURSOS OSEOS}

A fin de examinar estas referencias dentro del contexto de las fechas a las que pertenecen los restos óseos, es necesario seleccionar aquéllosque corresponden aproximadamente al período en que se llevó a cabo el contacto con los europeos y que coinciden con la zona de la descripción. Está por demás decir que no hubiera sido fructífero comparar las narraciones acerca de los indígenas de la región del cabo con los huesos de los primeros pobladores de La Jolla, quienes 
son originarios de la costa del Pacífico del sur de California(Estados Unidos). Tres zonas culturales son examinadas: la región del cabo, el desierto central y la región norte.

\section{COLECCIONES DEL MUSEO}

De todos los miles de personas que habitaron la península en los últimos milenios, existen sólo aproximadamente 100 individuos representados a través de restos óseos en todos los museos del mundo.

La región del cabo. La colección más grande se encuentra en el Instituto Nacional de Antropología e Historia de la ciudad de México. Esta colección fue hallada en la región del cabo por el difunto William C. Massey, de la Universidad de California en Berkeley. Más de 60 individuos se manifiestan en aproximadamente 43 tumbas (Massey, 1955); muchos de éstos son fragmentos y están en condiciones deficientes, y sólo 17 hombres y 10 mujeres están suficientemente completos como para que se efectúe un análisis métrico (Tyson, 1977: 169).

El Museo Lowie de Antropología de la Universidad de California en Berkeley, posee varios fragmentos de especímenes. El Museo Nacional de Estados Unidos, Institución Smithsoniana, tiene dos ejemplaresen buenas condiciones. El Museo del Hombre en París, aloja pequeñas colecciones adquiridas por Ten Kate y León Diguet, contándose con 26 muestras aproximadamente (Tyson, 1976).

El desierto central. La mejor colección de material proveniente del desierto central fue descubierta por el naturalista Edward Palmer en 1887, y se encuentra en el Museo Nacional de los Estados Unidos (Wilson, 1890: 123-128). La colección fue traída de una cueva fúnebre, localizada en bahía de Los Angeles al este de la costa de la península (Massey, 1961: 336-363), y la muestra consiste en los restos de cinco adultos y tres niños (Noble, 1973).

El Museo del Hombre en París, posee un esqueleto procedente del desierto central, mientras que el Museo del Hombre de San Diego cuenta con tres esqueletos, de los cuales uno es de la cultura de La Jolla, una de las primeras de esta zona.

La región norte. El Museo del Hombre de San Diego cuenta con tres ejemplares de los últimos pobladores de la cultura yumana y muchos de la cultura de La Jolla.

\section{FECHAMIENTO}

Con el fin de comparar las tres muestras, es necesario establecer su asociación arqueológica y su localización en el tiempo.

Sur. La cultura de Las Palmas (finales de la prehistoria) de aproximadamente $580 \pm 50$ años antes del presente (Tyson, 1977: 168). 
Central. La cultura Comondú (finales de la prehistoria y principios de la historia) (Massey, 1966: 50).

Norte. Cultura yumana (finales de la prehistoria y principios de la historia).

Cultura de Las Palmas. Los restos fúnebres secundarios pintados de rojo de la región del cabo, Massey los adjudicó a la cultura de Las Palmas (Massey, 1966: 47-59 y 1955: 47-59). Los utensilios perecederos asociados con los entierros incluyen flechas de madera, redes con nudos, recipientes elaborados con corteza de palma, objetos de madera dura en forma de rótula con dientes de tiburón en la orilla, y adornos de concha de ostión grabados en forma de pez (Massey, 1966: 47-49). Las fibras vegetales de una de las tumbas secundarias de cabo San Lucas, fueron fechadas aproximadamente entre 530 y 630 años antes de la época actual (Tyson, 1977: 168). Esto demuestra que la gente de la cultura de Las Palmas vivía en la región del cabo entre 100 a 200 años antes de tener contacto con los europeos. Las narraciones históricas no mencionan las costumbres fúnebres de la gente de Las Palmas, mismas que quizá hayan sido reemplazadas por la cremación antes del contacto. Durante su estancia en La Paz, el padre Nápoli escribió que sólo los valientes que morían en batalla eran enterrados, los otros eran cremados (Moriarty \& Smith, 1970: 42).

La cultura Comondú. Massey (1966: 50) delimitó el territorio de la cultura Comondú, que abarca desde un poco al sur de Comondú ( $26^{\circ}$ latitud Norte) hasta bahía de Los Angeles ( $28^{\circ} 55^{\prime}$ latitud Norte); la cual, de manera general corresponde a la región del desierto central. Transitoriamente, la cultura Comondú es asociada con el final de la fase de la prehistoria y el principio de la historia de la parte central de la península. Las características de los utensilios son: tazones poco profundos y piedras lisas para moler acompañadas de la piedra-mano, cestos poco profundos de base atada tejidos en espiral, pequeñas puntas de flecha triangulares de piedra caliza y de obsidiana con orillas aserradas, ganchos de pitahaya hechos de cuerdas de maderadura sujetos a un palo de caña, mallas para carga y redes para el cabello elaboradas con la técnica de nudos cuadrados, capas hechas de cabello humano, y pipas de piedra en forma de tubo (Massey, 1966: 51). Los objetos encontrados junto con los restos óseos de bahía de Los Angeles señalan ese lugar como perteneciente a la cultura Comondú.

La cultura yumana. Las características arqueológicas de la cultura yumana (no debe confundirse con la clasificación lingüistica yumana) del sur de California y del norte de Baja California, incluyen alfarería y pipas de barro cocido, cestería con bases de atadura en espiral, dardos de puntas triangulares, arco y flecha, palas de madera y palos para cavar, pipas cilíndricas de piedra y tubos de succión, morteros de piedra, vasijas de poco fondo, metates junto con su mano, pinturas rupestres, tablas de madera pintada, viviendas abandonadas y cremaciones (Hedges: comunicación personal). Las tumbas de tres mujeres del norte de la región son una muestra que manifiesta la influencia española, ya que todas fueron enterradas en posición horizontal y completamente rectas; además, porque dos de ellas tenían materiales de la cuitura europea así como materiales aborígenes (registros del Museo del Hombre en San Diego). 


\section{LAS CARACTERISTICAS FISICAS COMO MUESTRA DEL ANALISIS METRICO}

Los métodos y las clasificaciones de Martín y Saller (1956) fueron usados para obtener mediciones e indicadores. La fórmula de Pearson(Hooton 1946: 739) fue utilizada para calcular la capacidad craneana y las reconstrucciones de la estatura fueron efectuadas a través de las tablas de Genovés (1967: 67-78).

La región del cabo. La muestra consiste en 17 hombres y 10 mujeres (cuadro 1). Ambos sexos poseen cráneos hiperdolicocéfalos (cabeza muy larga). Las caras son anchas con aberturas nasales amplias. Las mujeres presentan mayor prognatismo (destacando caras más chicas) que los hombres (Tyson, 1977: 169).

La estatura promedio estimada para los hombres es de $164.7 \mathrm{~cm}$ (rango de 159.0 a $172.5 \mathrm{~cm}$ ) y $159.7 \mathrm{~cm}$ para las mujeres (rango de 152.0 a $168.5 \mathrm{~cm}$ ) (Tyson, 1977: 169-170).

La región del desierto central. La muestra medible consiste en tres hombres y tres mujeres. Los hombres son clasificados como dolicocéfalos (cabeza larga) y las mujeres como mesocéfalos (tamaño intermedio). Las caras son de medianas a anchas con aberturas nasales amplias. Presentan poco prognatismo. (Noble, 1973: 173).

Había sólo dos especímenes cuya estatura pudo ser medida: un hombre de $162 \mathrm{~cm}$ y una mujer de $149 \mathrm{~cm}$ (Noble, 1973: 116).

La región norte. La muestra consiste en tres mujeres (Noble, 1973: 158). Estas se clasificaron como braquicéfalas, hiperbraquicéfalas y ultrabraquicéfalas (de cabeza amplia, muy amplia y extremadamente amplia). El ancho de la cara varía de intermedia a amplia, y la abertura nasal es de media a amplia. Dos de las mujeres presentan prognatismo (Noble, 1973: 177-178).

La reconstrucción de la estatura para las dos mujeres medidas es de 151 y 153 cm (Noble, 1973: 164).

\section{RESUMEN}

Utilizando ambos, los relatos históricos y el análisis métrico actual de los restos óseos, surge una más completa descripción de la población prehistórica de la península.

La región del cabo. Esta gente era relativamente alta en comparación con otros grupos de indígenas observados en el territorio central de México y con los mismos exploradores (muchos de los primeros exploradores europeos eran relativamente bajos de estatura en comparación con los hombres de la época actual). El color de la piel variaba según se tratara de grupos del interior o de la costa, ya que por la exposición a los rayos solares, los de la costa eran de piel más obscura. El cabello era lacio, negro y lo usaban largo. La forma de la cabeza 


\section{CUADRO 1.}

\begin{tabular}{lccccc}
\hline INDICES & \multicolumn{2}{c}{$\begin{array}{c}\text { Región del cabo* } \\
\text { Hombres }\end{array}$} & Mujeres & Desierto central** & Región norte** \\
Mujeres & Mujeres \\
\hline $\begin{array}{l}\text { Indicador } \\
\text { craneano }\end{array}$ & $66.1(17)$ & $66.2(10)$ & $72.9(3)$ & $77.4(2)$ & $87.2(3)$ \\
$\begin{array}{l}\text { Indicador } \\
\text { facial } \\
\text { superior }\end{array}$ & $50.7(15)$ & $51.5(10)$ & $50.0(3)$ & $49.2(3)$ & $48.1(3)$ \\
$\begin{array}{l}\text { Indicador } \\
\text { nasal }\end{array}$ & $51.3(15)$ & $53.4(10)$ & $52.1(3)$ & $55.8(3)$ & $50.2(3)$ \\
$\begin{array}{l}\text { Indicador } \\
\text { de } \\
\text { mandibula }\end{array}$ & $102.2(15)$ & $103.0(9)$ & $100.3(3)$ & $98.9(2)$ & $102.2(3)$ \\
\hline
\end{tabular}

* Tyson, Rose. "Human Skeletal Material from the Cape Region of Baja California, Mexico: The American Collection". Journal de la Société des Americanistes. Vol. 64. París, 1977.

** Noble, Rose A. Physical Anthropology of Baja California. M.A. Thesis Department of Anthropology, San Diego State University, San Diego, California, 1973.

( ) Señala el número de individuos de la muestra.

era larga y angosta con amplios huesos en los pómulos y nariz ancha. La piel arrugada es muestra de una avanzada edad, o quizá se debe a alguna tarea que requería de un período prolongado de exposición a los fuertes rayos solares más que a la vejez. En un estudio de McCown(Tyson, 1979: 19) de los restos de 16 hombres y 10 mujeres de la colección de Massey que se encuentra en el Instituto Nacional de Antropología e Historia, sólo dos individuos pudieron ser clasificados en la categoría de más de 50 años de edad.

Región desierto central.Son pocas las descripciones aceptables de los indígenas del desierto central; no obstante, éstas sugieren una estatura más baja comparada a la de aquéllos de la región del cabo. La evidencia ósea también sugiere población de estatura baja.

Región norte. Los relatos históricos de North y Chittenden sólo mencionan a los hombres como relativamente altos. La evidencia física es escasa, ya que la cremación fue el método predominantemente utilizado para deshacerse de los muertos. Sólo tres mujeres - probablemente del grupo kumiai- del período postespañol han sido examinadas. Esta pequeña muestra indica mujeres de cabeza amplia con ligero prognatismo y estatura baja.

Comparando las tres culturas contemporáneas, la forma de la cabeza cambia desde muy angosta en la región del cabo, hasta intermedia en el desierto central, y amplia en la región norte. 
Aunque las muestras son pequeñas, las reconstrucciones indican que la gente de la región del cabo era más alta, y la del desierto central más baja. No se dispone de restos óseos para reconstruir la estatura masculina en la región norte. Los relatos históricos indican que los hombres eran relativamente altos. 


\section{BIBLIOGRAFIA}

ASCHMANN, Homer. 1966. "The Natural and Human History of Baja California from Manuscripts by Jesuit Missionaries". Baja California Travels Series, № 7. Dawson's Book Shop, Los Angeles.

1967. The Central Desert of Baja California: Demography and Ecology. Menessier Publishing Co., Riverside, California.

BAEGERT, Johann Jacob. 1952. Observations in Lower Califormia. M.M. Brandenburg and Carl L. Baumann, trans. and eds. Berkeley: University of California Press.

BELDING. L. 1985. "The Pericue Indians". The West American Scientist. Vol. 1 , tomo 4.

BURRUS, Ernest J: 1954. Kino Reports to Headquarters. Institutum Historicum Societatis Jesu. Roma.

1971. "Juan María de Salvatierra, S.J.: Selected Letters About Lower California”. Baja California Travels Series. № 25. Dawson's Book Shop. Los Angeles.

CHITTENDEN, Newton H. 1901. “Among the Cocopahs”. Land of Sunshine. Vol. 14, tomo 3.

CLAVIJERO, Francisco Xavier. 1971. The History of (Lower) California. Sara E. Lake, trans., and A. Gray, ed. Manessier Publishing Co. (publicado por vez primera en 1937 por la Universidad de Stanford) Riverside, California.

DIGUET, León. 1898. "Rapport sur une Mission Scientifique dans la BasseCalifornie”. Extrait des Nouvelles Archives des Missions Scientifiques. Tomo IX.

1905. “Anciennes Sepultures Indigenes de la Basse-Californie Meridionale”. Journal de la Société des Americanistes de Paris. (New Series).

GABB, William. 1867. Cochimi and Kiliwee Comparative Vocabulary. Bureau of American Ethnology, Catalog of Manuscripts, № 1147. National Anthropological Archives, Smithsonian Institution.

GENOVES, Santiago. 1967. "Proportionality of the Long Bones and their Relation to Stature among Mesoamericans". American Journal of Physical Anthropology.

HOHENTHAL, W.D., Jr. 1951. The Mountain Tribes of Northern Baja California, México. Paper for Anthropology 299, University of California, Berkeley.

HOOTON, E.A. 1946. Up from the Ape. The MacMillan Co. New York.

MARTIN, Rudolf and Karl Saller. 1956. Lehrbuch der Anthropologie in Systematischer Darstellung. Stuttgart: Gustav Fischer Verlag.

MARTINEZ, Pablo L. 1956. Historia de Baja California. Libros Mexicanos. México, D.F.

MASSEY, William C. 1955. Cultural History in the Cape Region of Baja California. Unpublished Ph. D. Dissertation, University of California, Berkeley.

1966. "Archaeology and Ethnohistory of Lower California". en: Handbook of Middle American Indians. Vol. 4. Robert Wauchope, Ed. Austin. University of Texas Press. 
MASSEY, William C. and Carolyn M. Osborne. 1961. "A Burial Cave in Baja California: The Palmer Collection, 1887". University of California of Anthropological Records. Vol. 16, tomo 8.

MEIGS, Peveril III. 1939. "The Kiliwa Indians of Lower California". IberoAmericana 15. University of California Press.

MORIARTY, James Robert, III, and Benjamin F. Smith, trans. and eds. 1970. "The Cora Indians of Baja California: The Relation of Father Ignacio Maria Napoli, S.J., September 20, 1721". Baja California Travels Series, № 19. Dawson's Book Shop Los Angeles.

NOBLE, Rose A. 1973. Physical Anthropology of Baja California. M.A. Thesis, Department of Anthropology, San Diego State University, San Diego, California.

NORTH, Arthur W. 1908. "The Native Tribes of Lower California". American Anthropologist. Volumen 10.

ROGERS, Woods. 1966. "A Description of California by Captain Woods Rogers, an Englishman, and of his Voyage to the South-Sea in the Year 1710". In: A Natural and Civil History of California. Vol. I, by Miguel Venegas. March of America Facsimile Series, № 38. Ann Arbor: University Microfilms, Inc.

SALES, Luis, O.P. 1956. "Observations on California, 1772-1790". Charles N. Rudkin, trans. and ed. Early California Travels Series, № 37. Glen Dawson. Los Angeles.

SHELVOCKE, George. 1928. A Voyage Round The World. Casell and Company, Ltd. London (publicado por primera vez en 1726).

SIMPSON, Lesley Byrd, trans. and ed. 1961. Journal of José Longinos Martínez: Notes and Observations of the Naturalist of the Botanical Expedition in Old and New California and the South Coast, 1791-1792. John Howell-Books. San Francisco.

TEN KATE, Herman. 1883. "Quelques Observations Ethnograpiques Recueillies dans la Presqu'ile Californienne et en Sonora”. Revue d'Ethnographie. Vol. 2, tomo 4.

1884. "Materiaux pour Servir a l'Anthropologie de la Presqu'ile Californienne". Bulletins de la Société l'Anthropologie de París (Third Series). Vol. 7.

TORQUEMADA, Juan de. 1966. "Narrative of the Voyage of Captain Sebastián Vizcaíno in the Year 1602, for Surveying the Outward or Western Coast of California on the South-Sea". En: A Natural and Civil History of California. Vol. I, by Miguel Venegas. March of America Facsimile Series, № 38. University Microfilms. Ann Arbor.

TYSON, Rose A. 1976. Museum Resources for the Study of the Physical Anthropology of Baja California. Documento presentado en la reunión anual de la Asociación Antropológica del Suroeste. San Francisco, abril.

1977. "Human Skeletal Material from the Cape Region of Baja California, Mexico: The American Collections". Journal de la Société des Americanistes. Vol. 64. París.

1979. An Analysis of Theodore McCown's Osteometric Data from the Cape Region of Baja California. Ponencia presentada en la reunión anual de la asociación antropológica del suroeste. Santa Bárbara, marzo 29.

WILSON, Thomas. 1890. Report on the Department of Prehistoric Anthropology in the U.S. 